\title{
Volume-Outcome in Cancer Surgery: Why has the Data Not Affected Policy Change?
}

\author{
Douglas B. Evans, MD and Susan Tsai, MD \\ Pancreatic Cancer Program, Division of Surgical Oncology, Department of Surgery, Medical College of Wisconsin, \\ Milwaukee, WI
}

It has been over 30 years since the report by Dr. Harold Luft and colleagues in the New England Journal of Medicine demonstrating an inverse relationship between hospital volume and mortality for selected surgical procedures. ${ }^{1}$ Almost 20 years later the Leapfrog Group, a consortium of large corporations and public agencies that purchase health care, suggested minimum volume standards for five high-risk surgical procedures as part of a broader, value-based purchasing initiative. ${ }^{2}$ However, use of volume as a quality metric remains controversial, as it is not a direct indicator of quality of care, but is often associated with quality and can be easily calculated. Over several decades, countless publications have strengthened the reproducible association of higher hospital volume and improved outcome (operative mortality), particularly with regard to pancreatic surgery. It is inescapable that some minimum experience (patient volume) is necessary for both the acquisition and maintenance of surgical skill and perhaps of equal or greater importance, the development of multidisciplinary teams which enhance all aspects of patient evaluation and treatment. Multidisciplinary teams likely impact multiple fundamental factors which affect outcomes including patient selection, perioperative treatment, and postoperative care. Not surprisingly, the benefit of high volume centers extends beyond the postoperative period and is associated with improved long-term patient survival. ${ }^{3}$ Patient volume and the clinical experience that it generates combined with outcome assessment (Hawthorne effect) makes for improved results. The published data in

\footnotetext{
(C) Society of Surgical Oncology 2014
}

First Received: 13 August 2014;

Published Online: 20 September 2014

D. B. Evans, MD

e-mail: devans@mcw.edu support of a volume-outcome relationship has resulted in some degree of regionalization of care in other countries, ${ }^{4,5}$ but a general lack of progress in the United States where a host of explanations have been brought forth to explain the lack of progress: patients may not want to travel or cannot afford the cost of travel; loss of experience for physicians at low volume hospitals (a concern of possibly greater consequence when dealing with patients who may require emergent surgery); financial implications of the possible loss of pancreatic cancer patients-likely not a significant concern for the surgery-related revenue (at low volume institutions), but to the extent that all pancreatic disease may leave (especially if lost from the hospital system), the downstream revenue for the system including infusion, pharmacy, and diagnostic imaging may be significant; and finally, the opinion that general surgeons are well trained in pancreatic and upper abdominal surgery and referral to specialty programs is unnecessary. In this issue of the Annals of Surgical Oncology, Drs. Swanson and colleagues report findings from the National Cancer Data Base, again supporting the volume-outcome (mortality) relationship for major pancreatic resection and they also find that 90-day mortality is twice that of 30-day mortality, even at high volume hospitals. ${ }^{6}$ The authors conclude that hospitals should be aware of their annual volume, the mortality rates at 30 and 90 days, and be benchmarked to high volume hospitals. The manuscript raises important questions: What other outcomes should be measured beyond 30-day mortality? With the excess of data supporting the volumeoutcome relationship, what barriers exist which prevent regionalization of care and how can we move the needle in the direction of policy change?

First, in the context of oncology, and especially for diseases such as pancreatic cancer which have a finite survival, it is clear that we need to measure other outcomes in addition to 30-day mortality. The patients included in the 
Swanson study underwent elective pancreatectomy (Table 2b. included pancreaticoduodenectomy, total pancreatectomy, or extended pancreatectomy-emergency operations of this kind are rare) and the authors demonstrated that the safety of elective pancreatic resection is not accurately reflected in 30-day mortality. We are left to conclude that 90-day mortality reflects treatment-related complications because disease-related death within 90 days would be very uncommon even for pancreatic cancer, assuming that the patient was deemed an acceptable operative candidate. A cumulative 90-day treatment-related mortality rate of $8.0 \%$ is unacceptable when one considers the very modest potential for treatment-related cure (further comment below). That said, pancreatic cancer represents a unique opportunity to further refine outcome criteria to more accurately reflect the safety and oncologic benefit of surgery. In addition to 90-day mortality, such criteria would include rates of reoperation, readmission, and length of hospital stay. Beyond assessment of the safety of surgery, we need to assess the impact of surgery on patient-centered outcomes including discharge destination (home vs nursing facility) and performance status (to include independence with self-care) at 90 and perhaps 180 days. These latter statistics would account for the higher risk operations which may have been associated with surgery-related morbidity but resulted in a very good intermediate-term outcome. For patients who undergo pancreatic surgery for cancer, survival at one, two, and three years should be measured. It is time to acknowledge that the ability to do the operation safely does not justify its performance; hospitals (and physicians) should also be accountable for the impact of elective surgery on subsequent patient-centered outcomes (functional independence and survival duration), especially when the recommended operations have such defined risks for morbidity and mortality and options exist for non-surgical therapies.

Assuming we follow the recommendations of Swanson and colleagues and measure more than 30-day surgeryassociated mortality, what is the impact of another outcome measurement if we fail to acknowledge the reproducible volume-outcome data generated over the past decades and translate it into health policy? Patients with pancreatic cancer continue to be operated upon in low volume hospitals resulting in unnecessary morbidity/mortality and inferior survival durations-what is responsible for the inferior outcome and why does it continue? Optimal care of the patient with pancreatic cancer requires a small village. The village includes a multidisciplinary team of physicians who have achieved consensus on both staging and stagespecific treatment (on- and off-protocol) and a support network of advanced care practitioners, nurses, and referral specialists who facilitate initial evaluation and the management of treatment-related toxicities. The "10,000hour rule" as described by Malcom Gladwell in his book Outliers, examined the time required to achieve mastery in a field. ${ }^{7}$ Although he referred to largely non-medical fields, practice makes perfect in more than athletics and music; medicine is not an exception. A reasonable volume of patient referral is required to both provide the necessary programmatic experience and to justify (pay for) the village of support personnel-in the absence of such patient volume, disease-specific specialists/programs do not exist. The management of patients with localized pancreatic cancer could be regulated similar to trauma and the emerging policy changes in pediatric surgery. Patients can be referred for initial staging, possible biopsy, and biliary stenting if necessary and the multidisciplinary team can then suggest which aspects of care can be delivered in the patient's local community. Newly diagnosed patients who are candidates for anti-cancer therapy (rather than best supportive care) should be offered referral to a specialty center and this discussion documented in the medical record. Such documentation could serve as a metric for reimbursement. With regard to surgery, whether or not a surgeon in a given hospital feels qualified to remove part or all of the pancreas is not the question-this misguided focus on the surgeon has allowed the debate to continue. If the resources to manage the disease (which clearly require some reasonable volume of patients to justify their financial existence) do not exist, the patient should receive operative care at a larger specialty/referral center-the surgeon is just one piece of this debate which needs to be depersonalized.

In light of the mountain of evidence in support of avoiding pancreatic surgery at low volume hospitals, why does this continue? Some possible explanations: 1. Some surgeons disagree with the data and feel they can perform the operation as well as those who do it weekly-answered above-even if this is so, a low volume hospital does not have the other members of the team necessary to optimize patient selection for surgery, incorporate contemporary treatment sequencing to include medical and radiation oncology, and the preoperative and postoperative expertise and supportive care that comes with practice. 2. Hospital systems receive a significant contribution margin for the care of each patient with pancreatic cancer and "leakage" from the system (to a higher volume institution) is actively discouraged even for the minority of patients who require surgery. As physicians have become increasingly employed by multispecialty practice groups or hospital systems and the employment platform includes wRVU requirements/incentives, this has become a major concern. Physicians may be pressured to treat a patient(s) who they know would be better managed at another institution. 3 . Physicians and those in hospital administration simply 
remain uninformed or choose to personalize a principled debate by insisting that volume does not matter and efforts to regionalize care are simply inappropriately restricting the practice of medicine. This, if combined with a somewhat fatalistic attitude toward the care of pancreatic cancer, can pose a formidable argument in support of treating the patient close to home even if not "in the right place at the right time". 4. The practice of general surgery is in conflict, and for non-fellowship trained general surgeons, the conflict is the loss of much of what they do (or used to do) to specialty-trained surgeons. This conflict often contains a high level of emotion, has generated considerable debate on how we train surgeons, and at present, the patient is left in the middle. Non-fellowship trained general surgeons are granted hospital privileges and are often expected to perform pancreatic surgery for cancer after an experience that is usually in the low single digits as a resident-this is simply wrong. In urban environments, general surgery largely does not exist and has been replaced by acute care, emergency and trauma surgery. Most all other areas (including acute care/trauma surgery) have evolved specialty (fellowship) training for the simple reason that volume does matter-both for the individual surgeon and to allow for needed program development (creation of the village). The relationship of hospital volume to patient outcome is not lost on the educated consumer who will seek out high volume referral centers. The training of our residents, as well as system-wide hospital policy, will need to adapt to this reality.

For now, what can we do to move the needle in the right direction? Perhaps baby steps forward: 1 . As suggested by Dr. Swanson, elective pancreatic surgery should not be performed at hospitals with an annual volume of less than 10 operations/year; this can be operationalized by restricting reimbursement. 2. We can do a better job of refocusing attention from "the case" to management of the patient and his/her disease. This paradigm shift should begin in residency and include a change in how we measure hospital outcomes to include performance status (at 90 and 180 days) and survival duration for all operated cancer patients. 3. Pressure applied to physicians to keep patients in "the system" rather than offer them referral to a center who can do it better should be forbidden and associated with a harsh financial penalty. We should all look upon the decades of volume-outcome data (all with the same findings and conclusions) and the lack of policy change as a huge failure. All future manuscripts examining this topic in surgery/oncology should be required to include recommended policy change; transforming data-driven principled debate to patient management is long overdue.

\section{REFERENCES}

1. Luft HS, Bunker JP, Enthoven AC. Should operations be regionalized? NEJM 1979;301:1364-9.

2. Birkmeyer JD, Finlayson EVA, Birkmeyer CM. Volume standards for high-risk surgical procedures: potential benefits of the Leapfrog initiative. Surgery 2001;130:415-22.

3. Birkmeyer JD, Sun Y, Wong SL, Stukel TA. Hospital volume and late survival after cancer surgery. Ann Surg 2007;245:777-83.

4. deWilde RF, Besselink MGH, van der Tweel I, et al. Impact of nationwide centralization of pancreaticoduodenectomy on hospital mortality. Br J Surg 2012;99:404-10.

5. Sonnenday CJ, Birkmeyer JD. A tale of two provinces: regionalization of pancreatic surgery in Ontario and Quebec. Ann Surg Oncol 2010;17:2535-36.

6. Swanson RS, Pezzi CM, Mallin K, Loomis AM, Winchester DP. The 90-day mortality after pancreatectomy for cancer is double the 30-day mortality: more than 20,000 resections from the National Cancer Database. Ann Surg Oncol. 2014. doi:10.1245/s10434-0144036-4.

7. Gladwell M. Outliers. New York: Little, Brown and Co; 2008. 